\title{
PREFERENTIAL POSITRON ANNIHILATION IN BINARY ALLOYS
}

\author{
J. Kuriplach ${ }^{a}$, E. DrYzeK ${ }^{b}$, J. DryzeK ${ }^{b}$, AND M. ŠoB ${ }^{c}$ \\ ${ }^{a}$ Department of Low Temperature Physics, Charles University \\ V Holešovičkách 2, 18000 Prague, Czech Republic \\ ${ }^{b}$ Institute of Nuclear Physics, Radzikowskiego 152, 31-342 Kraków, Poland \\ ' Institute of Physics of Materials, Academy of Sciences of the Czech Republic \\ Žǐkova 22, 61662 Brno, Czech Republic
}

The effect of preferential positron annihilation at individual constituent atoms in binary alloys is examined theoretically and experimentally. In particular, the results of recently studied alloys $\mathrm{Fe}-\mathrm{Al}$ and $\mathrm{Mg}-\mathrm{Cd}$ together with new preliminary results for the $\mathrm{Sn}$-In system are discussed and summarised. In the case of the Sn-In system, the concentration dependencies of positron annihilation rates and Doppler broadening spectra are analysed.

PACS numbers: $78.70 . \mathrm{Bj}, 71.60 .+\mathrm{z}$

\section{Introduction}

If two distinct elements form an alloy, it is interesting to examine how the positron annihilation characteristics depend on those observed in pure constituents. The probability of positron annihilation at a given place in a solid depends on both the positron and electron density at this place. In the case of an alloy, both positron and electron distributions differ from those of pure elements, even if the crystal structure and atomic volume is the same. In the case of the positron annihilation rate $\lambda$, it is natural to expect that the concentration dependence for an $\mathrm{A}_{1-x} \mathrm{~B}_{x}$ alloy in an ideal case, when $\mathrm{A}$ and $\mathrm{B}$ electronic densities superpose, should obey a relation

$$
\lambda_{\text {id }}(x)=\lambda_{\mathrm{A}}(1-x)+\lambda_{\mathrm{B}} x .
$$

In this relation $\lambda_{A}\left(\lambda_{B}\right)$ denotes the annihilation rate for the pure element $A(B)$, respectively.

Due to the charge redistribution, the positron annihilation rates characteristic of atoms of individual constituents of the alloy may differ from their values for pure constituents (elements). If such a characteristic rate for atoms of the A-type is larger than in the elemental solid $\mathrm{A}$, we are talking about the preferential positron annihilation (PPA) at A sites [1]. To be more specific, the annihilation rate for a real alloy may be written as

$$
\lambda(x)=\lambda_{\mathrm{A}}(x)+\lambda_{\mathrm{B}}(x),
$$


where the functions $\lambda_{\mathrm{A}}(x)$ and $\lambda_{\mathrm{B}}(x)$ describe the contributions to the total annihilation rate from $\mathrm{A}$ and $\mathrm{B}$ atoms, respectively. If

$$
\lambda_{\mathrm{A}}(x) /(1-x)>\lambda_{\mathrm{A}} \quad \text { or } \quad \lambda_{\mathrm{B}}(x) / x>\lambda_{\mathrm{B}},
$$

the PPA occurs at A sites or $\mathrm{B}$ sites (for a given concentration $x$ ). Thus, we define the positron annihilation rate characteristic of the $A(B)$ constituent by the ratio $\lambda_{\mathrm{A}}(x) /(1-x)\left[\lambda_{\mathrm{B}}(x) / x\right]$, respectively. The meaning of these relations will be demonstrated later. One may expect that the "enhancement" of the annihilation rate at A sites will result in the lowering of the rate at B sites.

By analogy, the momentum distribution $\varrho$ of annihilation photons for the above mentioned ideal alloy should be given by the expression

$$
\lambda_{\mathrm{id}}(x) \varrho_{\mathrm{id}}(x, p)=\lambda_{\mathrm{A}} \varrho_{\mathrm{A}}(p)(1-x)+\lambda_{\mathrm{B}} \varrho_{\mathrm{B}}(p) x,
$$

where $\varrho_{\mathrm{A}}$ and $\varrho_{\mathrm{B}}$ are respective momentum distributions for $\mathrm{A}$ and $\mathrm{B}$ elements, which are supposed (together with $\varrho_{\mathrm{id}}$ ) to be normalised to the same (integral) value. The integration of Eq. (4) over momentum $(p)$ then leads to Eq. (1).

In our previous papers we studied preferential positron annihilation in $\mathrm{Fe}-\mathrm{Al}$ [2] and $\mathrm{Mg}-\mathrm{Cd}$ [3] systems. Here we present preliminary theoretical and experimental results for $\mathrm{Sn}$-In alloys and discuss them together with results previously found in $\mathrm{Fe}-\mathrm{Al}$ and $\mathrm{Mg}-\mathrm{Cd}$. Features common for these three systems are pointed out.

\section{Methods}

\subsection{Theoretical calculations}

To simulate Sn-In alloys, we employed the supercell approach. The electronic structure of selected alloys was calculated using the linear muffin-tin orbital (LMTO) method [4]. The basis functions included $s, p, d$, and $f$ orbitals (last two being downfolded [4]). The radii of atomic spheres for Sn and In atoms were chosen to be equal in all alloys. The zero positron density limit and local density approximation for positrons were utilised [5]. The electron-positron correlation potentials and enhancement factors were used according to Boroniski and Niemi- . nen [6] and Barbiellini et al. [7], last approach being based on the generalised gradient approximation. Corresponding calculations are further denoted as BN and GGA calculations, respectively. The positron annihilation rate (inverse of the positron lifetime $\tau$ ) may be calculated using the formula [5]

$$
\lambda=1 / \tau=\int\left|\psi_{p}(r)\right|^{2} n_{-}(r) \gamma\left[n_{-}(r), \nabla n_{-}(r)\right] \mathrm{d} r,
$$

where $\psi_{\mathrm{p}}$ is the positron wave function, $n_{-}$stands for the electron density, and $\gamma$ is the electron enhancement factor mentioned above. In the case of the GGA approach, the enhancement factor depends not only on $n_{-}$but also on its gradient [7].

\subsection{Experiment}

The phase diagram of the Sn-In system is rather complicated [8]. There are only a few concentration regions where some well defined phases exist. For other concentrations a mixture of phases can be observed. Both Sn and In have 
tetragonal structures. We are further interested in the $\gamma$-phase, which is simple hexagonal, and $\beta$-phase having a tetragonal structure. These two phases exist in the approximate composition ranges of $15-22$ and $73-89$ at.\% In, respectively. For the sample preparation procedure we refer the reader to [8]. We studied samples with the following atomic concentrations of In: $0,13,15,18,20,75,81,86$, and $100 \%$.

Lifetime measurements were carried out on a fast-fast spectrometer with NE111 plastic scintillators. The time resolution achieved (FWHM) was about $300 \mathrm{ps}$ for ${ }^{22} \mathrm{Na}$ source. The lifetime spectra were analysed using the program LT [9]. Doppler broadening spectra were collected using a coincidence spectrometer. A high-purity Ge detector (energy resolution $1.25 \mathrm{keV}$ at $511 \mathrm{keV}$ ) and $\mathrm{NaI}(\mathrm{Tl})$ scintillator detector were used for the coincidence detection of annihilation events. In this case, we employed a ${ }^{68} \mathrm{Ge}$ source to reduce the background in measured spectra. The peak to background ratio was about 2000:1. The remaining background in spectra was subtracted and spectra were appropriately normalised.

\section{Results for Sn-In alloys}

First we examine the positron lifetime measurements. In Fig. 1 positron annihilation rates obtained from experiment are plotted for all samples. We also plot a straight (solid) line which represents the ideal case according to Eq. (1). In the inset we also show the dependence of the atomic volume on the In concentration. We can see that the difference of volumes of $\mathrm{Sn}$ and In is very small (about $3 \%$ ). The decrease in the atomic volume is accompanied by a decrease in the number

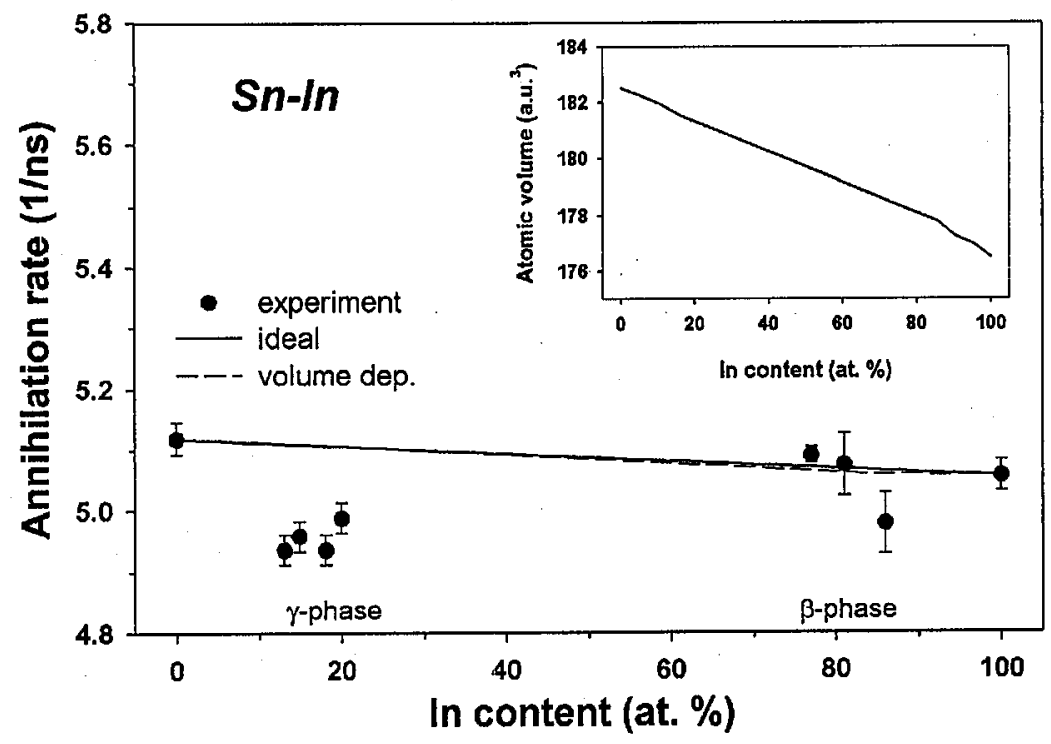

Fig. 1. Experimental positron annihilation rates for Sn-In alloys. The meaning of the solid and dashed curves is explained in the text. The inset shows the dependence of the atomic volume on the In content. 
of conduction electrons, which should result in an almost constant dependence of the positron annihilation rate. Indeed, there is a very small difference between the Sn and In annihilation rates (about $1 \%$ ).

In our previous studies $[2,3]$ we emphasised the effect of the volume dependence of the annihilation rate when evaluating the PPA. Here we use the well known Brandt and Reinheimer formula [10] $\left(\lambda=2+134 n_{-}\left(\lambda\right.\right.$ is in $\mathrm{ns}^{-1}$ and $n_{-}$in atomic units, a.u.)) to estimate the volume dependence of the annihilation rate in Sn-In alloys (see [2,3] for details). The result of this procedure is also shown in Fig. 1 (dashed curve). This curve almost coincides with the straight line discussed above. Thus in the case of the Sn-In system, the volume effect appears not to be important. On the other hand, the annihilation rates for samples in the $\gamma$-phase lie apparently below the both curves discussed. Such a deviation is not confirmed by theoretical calculations (see below) and it seems that some shallow positron traps may exist in these samples (see a detailed discussion in [8]), which, however, can hardly be deduced from the lifetime spectra as the one component lifetime analysis leads to a satisfactory description of the spectra measured. To have a more precise idea of the origin of the deviation observed, we would need to perform lifetime measurements for other Sn-rich alloys.

To examine the PPA in the Sn-In system, we performed a set of theoretical calculations at a constant atomic volume [2]. Here we choose the $\mathrm{Sn}$ atomic volume and expect that the results obtained are very similar for other volumes as the dependence of the volume on the concentration is rather weak (see the inset in Fig. 1). We calculated annihilation rates for $\mathrm{Sn}, \mathrm{Sn}_{3} \mathrm{In}, \mathrm{SnIn}_{3}$, and In systems

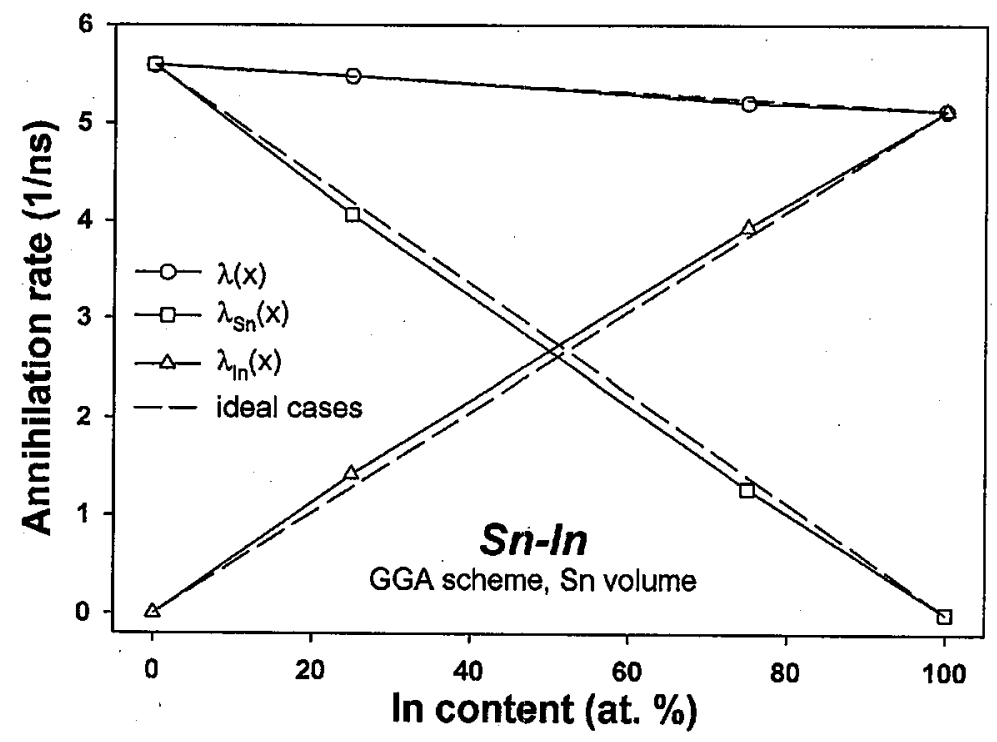

Fig. 2. Calculated positron annihilation rates for the $\mathrm{Sn}-\mathrm{In}$ system. The site resolved and total positron annihilation rates are shown. The atomic volume was fixed at the $\mathrm{Sn}$ value and the GGA calculational scheme was used. 
supposing crystal structures according to the phase diagram. For simplicity, the $\mathrm{Sn}_{3} \mathrm{In}$ alloy was taken to be based on the simple hexagonal lattice even if the concentration of In $(x=0.25)$ slightly exceeds the limit for the existence of the $\gamma$-phase (see Sec. 2.2). We should mention that the $\mathrm{Sn}$ and simple hexagonal lattices are not close packed structures and interstitial space is appreciable in contrast to, e.g., In, the structure of which is very close to the fcc structure. Therefore, the interpretation of the concentration dependence of $\lambda_{\mathrm{Sn}}(x)$ and $\lambda_{\mathrm{In}}(x)$ may not be straightforward because the division of the crystal among the Sn and In sites is not clear enough. In the following, we neglect this fact and do not include the empty spheres [4] when calculating the electronic structure for $\mathrm{Sn}$ and $\mathrm{Sn}_{3} \mathrm{In}$ systems. This may slightly influence the precision of such calculations.

Figure 2 shows the calculated dependencies $\lambda(x), \lambda_{\mathrm{Sn}}(x)$, and $\lambda_{\mathrm{In}}(x)$. The straight lines correspond to an ideal case without PPA. Here we present the results of GGA calculations only; in the case of BN, the particular rates differ slightly, but the conclusions drawn are the same (see below). Now we can use relations (3). It is clearly seen that the PPA occurs at In sites in the $\mathrm{Sn}-\mathrm{In}$ system as the $\lambda_{\mathrm{In}}(x)$ curve lies above the corresponding dashed line $\left(\lambda_{\operatorname{In}} x\right)$. In this respect, it is worth mentioning that there is a charge transfer from Sn to In sites. Even if it is rather small, it makes In sites more attractive for positrons than Sn sites.

Finally, in Fig. 3 we present examples of measured Doppler broadening spectra. In particular, we plot the curves which correspond to the difference: Sn spec-

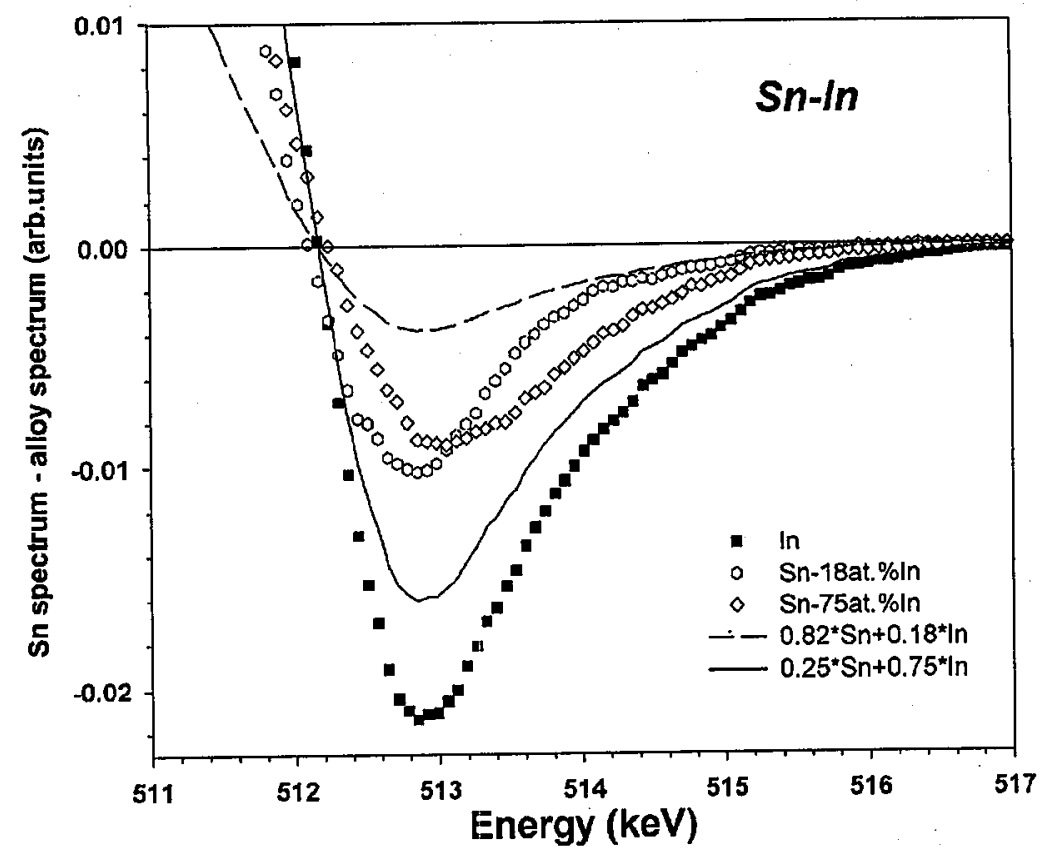

Fig. 3. Examples of differential Doppler broadening spectra for the Sn-In system. Hypothetical spectra for two In concentrations are also plotted (see the text for an explanation). 
trum - alloy spectrum. Here we omitted the multiplication by the annihilation rate (Eq. (4)) as the annihilation rates are nearly constant for all samples studied. For higher energies the Sn-In differential spectrum is positioned below zero, which corresponds to a larger core annihilation rate in In compared to Sn. As concerns the spectra of two alloys which we show in Fig. 3, we can see that they have similar "amplitudes", but their shapes slightly differ. In the figure we also plot hypothetical (differential) spectra created by the linear combination of $\mathrm{Sn}$ and In spectra for $x=0.18$ and $x=0.75$. At the first glance, one would say that there is a PPA at In sites for the Sn-18at.\%In alloy as the artificial spectrum for $x=0.18$ is positioned well above the spectrum for the Sn-18at.\%In alloy. Clearly, in the measured spectrum there is a larger contribution from In than in the ideal case. An opposite situation takes place for the second alloy, Sn-75at.\%In, i.e. the plot seems to indicate a PPA at Sn sites. These conclusions will require a further verification as we neglected the concentration dependence of the annihilation rate. One should also take into account possible positron traps at $\gamma$-phase samples we mentioned above.

\section{Discussion}

The preferential positron annihilation is an interesting effect reflecting the positron charge distribution in alloys. However, an experimental study of the PPA is not straightforward. The influence of the change of the atomic volume with the alloy composition was already mentioned above. Even if such an effect may be neglected (as in the Sn-In case) and the concentration dependence of the annihilation rate does deviate from the straight line (see $[2,3]$ ), it is still not clear at which sites the PPA occurs and a theoretical analysis of the contributions of alloy constituents to the total annihilation rate has to be done. Possible positron traps represent a further important point as their influence should be excluded from resulting experimental annihilation rates, which may not be always possible [8].

In the following chart we give a short summary of properties of all systems we studied theoretically so far with respect to the PPA.

\begin{tabular}{|c|c|c|c|c|}
\hline System & Electron configuration & PPA & Charge transfer to & Size of PPA (\%) \\
\hline $\mathrm{Fe}-\mathrm{Al}$ & $4 s 3 d-3 s 3 p$ & $\mathrm{Fe}$ & $\mathrm{Fe}$ & $+6.1, \quad-7.0$ \\
\hline $\mathrm{Mg}-\mathrm{Cd}$ & $3 s-5 s 4 d$ & $\mathrm{Mg}$ & $\mathrm{Cd}$ & $+2.0, \quad-2.5$ \\
\hline Sn-In. & $5 s 5 p-5 s 5 p$ & In & In & $-4.1,+4.1$ \\
\hline
\end{tabular}

Here the electron configuration refers to valence electrons and charge transfer to the sites where electrons are transferred. Thus, the alloys studied comprise metallic systems with various types of electronic structure. There are, however, some common features. First, it might be expected that the PPA occurs at the sites where electrons are transferred. This is valid for the $\mathrm{Fe}-\mathrm{Al}$ and $\mathrm{Sn}-\mathrm{In}$ systems. Nevertheless, for $\mathrm{Mg}-\mathrm{Cd}$ alloys the situation is opposite. In fact, we do not know yet the reason for this interesting effect.

Second, the column "size of PPA" denotes relative deviations $\lambda_{\mathrm{A}}(x)$ and $\lambda_{\mathrm{B}}(x)$ curves (obtained within the BN scheme) from the corresponding straight lines (see Fig. 2) taken at $x=0.5$. In general, it turns out that the PPA effect 
is rather small, at least at the systems examined. Especially a small effect can be seen for $\mathrm{Mg}-\mathrm{Cd}$ alloys, which is probably connected with the unexpected charge transfer effect mentioned above. We also note that for all systems investigated the sizes of PPA, in the sense explained above, have opposite signs for the constituents of a given alloy.

In the future, we also intend to investigate some $3 d$ and $4 d$ alloys where interesting effects concerning the PPA might be found [11].

\section{Acknowledgment}

We are grateful to O. Jepsen and O.K. Andersen for providing us with their LMTO computer program. We also thank M.J. Puska and T. Korhonen for the LMTO positron code.

\section{References}

[1] M.J. Stott, P. Kubica, Phys. Rev. B 11, 1 (1975).

[2] J. Kuriplach, M. Šob, C. Dauwe, Nukleonika 42, 153 (1997).

[3] E. Dryzek, J. Kuriplach, J. Dryzek, J. Phys., Condens. Matter 10, 6573 (1998).

[4] O.K. Andersen, O. Jepsen, M. Šob, in: Electronic Band Structure and Its Applications, Ed. M. Yussouff, Springer Verlag, Heidelberg 1987, p. 1.

[5] M.J. Puska, R.M. Nieminen, Rev. Mod. Phys. 66, 841 (1994).

[6] E. Boroński, R.M. Nieminen, Phys. Rev. B 34, 3820 (1986).

[7] B. Barbiellini, M.J. Puska, T. Korhonen, A. Harju, T. Torsti, R.M. Nieminen, Phys. Rev. B 53, 16201 (1996).

[8] E. Dryzek, J. Dryzek, Phys. Status Solidi A 144, K65 (1994).

[9] J. Kansy, Nucl. Instrum. Methods Phys. Res. A 374, 235 (1996).

[10] W. Brandt, J. Reinheimer, Phys. Lett. A 35, 109 (1971).

[11] E. Dębowska, Electronic Structure of Metal-Hydrogen Systems Studied by Positron Annihilation, Acta Universitatis Wratislaviensis, Wrocław 1992. 